\title{
A Canonical Transforming Growth Factor Beta-Dependent Signaling Pathway is Present in Peripheral Blood Cells of Cancer Patients with Skeletal Metastasis
}

\author{
Sunil Kadam ${ }^{1 *}$, Ann Louise Cleverly², Mark Farmen ${ }^{3}$, Janet Grondin', Yvelina Ivanova Cox ${ }^{1}$ and Michael Lahn ${ }^{4}$ \\ ${ }^{1}$ Translational Medicine, Eli Lilly and Company, Indianapolis, USA \\ 'European Early Phase Statistics, Eli Lilly and Company, Indianapolis, USA \\ ${ }^{3}$ Discovery and Development Statistics, Eli Lilly and Company, Indianapolis, USA \\ ${ }^{4}$ Oncology Business Unit, Eli Lilly and Company, Indianapolis, USA
}

\begin{abstract}
Background: Patients with advanced metastatic cancer often have elevated levels of circulating transforming growth factor beta (TGF- $\beta$ ) that is thought to stimulate receptor mediated signaling through phosphorylated SMAD transcription factors in peripheral blood mononuclear cells.

Methods: To identify this TGF- $\beta$-dependent gene expression profile in cancer patients, we first evaluated a multi-gene expression profile from ex-vivo treated PBMCs with TGF- $\beta 1$ stimulation. Change in expression, when challenged with a specific TGF- $\beta$ receptor type kinase inhibitor was then derived to be ligand and inhibitor specific. Once this profile was established, we examined its role in identifying the activation of a canonical TGF- $\beta 1$ signaling pathway in patient samples.

Results: We discovered a 37 gene sub-set where the expression profile from ex-vivo treated PBMCs was significantly associated with SMAD phosphorylation in cancer patients. We found significant correlation between the ex-vivo derived expression signature and circulating levels of TGF- $\beta 1$ in patient samples. Additionally, we report association between the expression profile and the presence of several plasma proteins in disease samples that are known to be concomitantly present with TGF- $\beta$ - dependent pathway activation.

Conclusion: An expression profile for TGF- $\beta 1$ cytokine mediated-signaling in cancer patients is identified, which may serve as a biomarker to measure the pharmacodynamic effect of TGF- $\beta$ inhibitors during clinical drug development and as marker of disease diagnosis.
\end{abstract}

Keywords: Peripheral blood mononuclear cells, Skeletal metastasis, Transforming growth factor beta, LY2157299 monohydrate, SMAD

Abbreviations: TGF- $\beta 1$ : Transforming Growth Factor Beta One; TGF- $\beta$ RI: Transforming Growth Factor Beta Receptor Type One; SMAD: Mothers Against Decapentaplegic Homolog; PBMCs: Peripheral Blood Mononuclear Cells; CPT: Cell Preparation Tubes; PBS: Phosphate Buffered Saline; MAIP: Multianalyte Immunoassay Panel

\section{Introduction}

The transforming growth factor beta (TGF- $\beta$ ) pathway plays a central role in a wide range of biological processes essential to embryonic development and tissue homeostasis [1].The canonical signaling event induced by TGF- $\beta$ superfamily members is initiated by ligand-mediated hetero-oligomerization of the serine-threonine kinase receptors, which phosphorylate signaling of Regulatory Smads (RSmads),such as Smad $2 / 3$ for TGF- $\beta$ s and Smad1/5/8 for Bone Morphogenetic Proteins (BMPs).The activated R-Smads then form a heterocomplex with a common Smad (Co-Smad or Smad4) and the complex is translocated into the nucleus, resulting in transcriptional regulation of abroad repertoire of target genes [2,3]. Other Inhibitory SMADs (I-SMADs) such as SMAD6 and SMAD7 generally inhibit BMP signaling and TGF- $\beta$ /activin signaling respectively and serve as negative regulators of TGF- $\beta$ dependent pathway signaling $[4,5]$. In addition to this canonical Smad-dependent signaling, TGF- $\beta$ and related ligands are also capable of activating small GTPases, mitogenactivated protein kinases, and phosphatidylinositol-3-kinase, initiating a so-called Smad-independent, non-canonical response [6].
TheTGF- $\beta$ superfamily of ligands is normally thought to contribute to both normal development and tumor suppression [5]. However during the course of cancer progression, the normal tumor suppression function of TGF- $\beta$ is often lost and its over-expression is associated with creating a local immunosuppressive environment that enhances tumor growth and exacerbates invasive and metastatic tumor cell behavior [2-4].

It has been previously reported that elevation in pSMAD levels in Peripheral Blood Mononuclear Cells (PBMCs) can be detected after ex-vivo stimulation with TGF- $\beta 1$ and this activation signal might represent a useful biomarker for evaluation of TGF- $\beta$ inhibitors [6-8]. Furthermore, this potential for cytokine induced phosphorylation of SMAD was found to be correlated with serum TGF- $\beta 1$ levels [6-8]. Separately, we have previously reported on the specificity of LY2157299 monohydrate kinase and its potent activity as an inhibitor of TGF- $\beta$

*Corresponding author: Sunil K Kadam, Translational Medicine, Eli Lilly and Company, Indianapolis, IN-46285, USA, Tel: + 317 966-2930; Fax: + 317 2761652; E-mail: s.kadam@lilly.com

Received October 24, 2013; Accepted November 19, 2013; Published November 21, 2013

Citation: Kadam S, Cleverly AL, Farmen M, Grondin J, Cox Yl, et al. (2013) A Canonical Transforming Growth Factor Beta-Dependent Signaling Pathway is Present in Peripheral Blood Cells of Cancer Patients with Skeletal Metastasis. J Mol Biomark Diagn 4: 153. doi:10.4172/2155-9929.1000153

Copyright: ( 2013 Kadam S, et al. This is an open-access article distributed under the terms of the Creative Commons Attribution License, which permits unrestricted use, distribution, and reproduction in any medium, provided the original author and source are credited 
Citation: Kadam S, Cleverly AL, Farmen M, Grondin J, Cox YI, et al. (2013) A Canonical Transforming Growth Factor Beta-Dependent Signaling Pathway is Present in Peripheral Blood Cells of Cancer Patients with Skeletal Metastasis. J Mol Biomark Diagn 4: 153. doi:10.4172/21559929.1000153

receptor type one (TGF- $\beta$ RI) mediated signaling using several pathway related protein markers including the phosphorylation of SMADs in tumor xenografts [9]. However, quantitative measurement of pSMAD from tumor derived TGF- $\beta$ requires separation of PBMCs from whole blood under conditions that minimize the effect of inherent platelet derived TGF- $\beta$; a significant pre-analytic source of variability. Secondly, this baseline variability in PBMC pSMAD measurement makes it difficult to estimate the residual post-translation SMAD phosphorylation capacity during an external cytokine challenge. We examined whether TGF- $\beta 1$ addition to PBMCs ex-vivo, was accompanied by changes in global gene expression and whether addition of a specific TGF- $\beta$ RI kinase inhibitor produced a specific changes in this expression pattern. We then reasoned that measurement of this change in gene expression might provide a handle to evaluate the influence of circulating TGF- $\beta 1$ in the blood of advanced cancer patients. Independent for TGF- $\beta$-related signaling, this approach of using microarray-based transcriptional profiling from peripheral blood, has been successfully applied to evaluate the diagnostic potential of transcript derived "signatures" in multiple sclerosis [10], chronic fatigue syndrome [11], sickle cell anemia [12], post-traumatic stress disorder [13], Huntington's disease [14] and acute respiratory distress syndrome [15]. Hence, while not novel, the approach can help with the dose determination of new TGF- $\beta$ inhibitors.

In parallel, over the last decade expression profiling procedures have been optimized with regard to the development of optimal standardized procedures for blood collection and subsequent RNA extraction [16,17]. Further improvement in reproducibility was possible with the assessment of precision of the various components of variability associated with sample handling and array data processing steps [18]. These advances have led to the recognition of expression signature based "intrinsic subtypes" in tissues and are now used to classify clinical specimens into phenotypic and prognostic categories [19-21].

We have investigated the role of PBMCs and whole blood, as potential "surrogates" for the development of biomarkers of TGF- $\beta$ dependent activation. The ex-vivo procedure developed for detection of the TGF- $\beta 1$ dependent stimulation of normal pSMAD in PBMCs was used to follow changes in global gene expression with LY2157299 monohydrate; a specific inhibitor of TGF- $\beta$ RI kinase. The measurement of ex-vivo transcript level changes was performed using RNA microarray and the resulting expression profiles derived from the ex-vivo assay were used to characterize expression patterns in the whole blood of advanced cancer patients. The underlying hypothesis assumes that patients with advanced cancer with elevated levels of plasma TGF- $\beta$ will produce a measurable change in the pattern of expression in PBMCs and mirror the ongoing biological processes during cytokine dependent cancer progression. We found that a TGF- $\beta$ stimulated and LY2 157299 reversed 37-gene expression profile, derived from the ex-vivo PBMC analysis, was also present in whole blood from cancer patients. Expression index of the composite genes from patient blood was significantly correlated with PF4 adjusted pSMAD in corresponding PBMCs and circulating levels of TGF- $\beta 1$ and other co-expressed factors known to be associated with canonical pathway activation.

\section{Material and Methods}

\section{Cell lines, compounds and reagents}

Recombinant human TGF- $\beta 1$ was purchased from R\&D Systems, (catalog \#240-B, Minneapolis, MN, USA). TRIzol cell lysis and RNA preservation reagent (Invitrogen, Carlsbad, CA) was used according to manufacturer's recommendations. Tempus tubes (Applied Biosystems, Carlsbad, CA) and PAX gene blood RNA tubes (PreAnalytiX/Qiagen, Feldbachstrasse CH-8634 Hombrechtikon, Switzerland) were used for collection of whole blood and RNA preservation and extraction was performed according to manufacturer's recommendations. LY2157299 monohydrate is a small molecule inhibitor of the TGF- $\beta 1$ receptor kinase [9,22-24]. Microarray analysis was performed using Affymetrix, HG-U133A human array chips (Affymetrix, Santa Clara, CA). Blood used to recover PBMCs for ex-vivo experiments was from normal subjects and was collected in Cell Preparation Tubes (CPT) from Becton Dickinson (Franklin Lakes, NJ, USA).

\section{PBMC separation and $e x$-vivo TGF- $\beta 1$ stimulation assay}

CPTs obtained from Becton Dickinson (Franklin Lakes, NJ, USA), containing a polyester gel and sodium citrate anticoagulant were used for blood collection and stored upright at room temperature for at least $2 \mathrm{~h}$ prior to sample collection to reduce temperature gradient shock during blood draw. Upon blood collection, the tube was immediately centrifuged in a clinical swinging bucket centrifuge at $800 \mathrm{~g}$ for $25 \mathrm{~min}$ at room temperature. The PBMC layer just above the gradient gel was gently resuspended into the separated upper plasma by slowly inverting the tube. Two $1.5 \mathrm{ml}$ fractions of the supernatant located above the polymer barrier were transferred into two $12 \times 75 \mathrm{~mm}$ polypropylene tubes. The polypropylene tubes were centrifuged at 1,600 g for $15 \mathrm{~min}$ and the supernatant was carefully removed. The PBMC pellet was resuspended in an equivalent volume of Phosphate Buffered Saline (PBS) with $1 \%$ Bovine Serum Albumin (BSA) recentrifuged at 1,600 $\mathrm{g}$ and washed once in PBS with 1\% BSA. Finally, pellets from two tubes per donor were resuspended in PBS with $1 \%$ BSA and pooled for treatment. A $200 \mu \mathrm{l}$ aliquot of the $3.0 \mathrm{ml}$ PBMC sample was used for each duplicate treatment. A stock was prepared and appropriate volumes were added to ligand containing tubes to achieve a final concentration of 1, 10, 50 and $100 \mathrm{pM}$. An equivalent volume of PBS with $1 \%$ BSA was added to the unstimulated PBMC control "vehicle" sample tube. Tubes were incubated at $37^{\circ} \mathrm{C}$ in a Dubnoff metabolic water bath (Precision Scientific, Chicago, IL, USA) for 4 hours with shaking at $60 \mathrm{rpm}$. Where stated LY2157299 monohydrate was added to achieve a $5 \mu \mathrm{M}$ concentration and the reaction incubated at $37^{\circ} \mathrm{C}$ for $5 \mathrm{~min}$ prior to addition of TGF- $\beta 1$. At the end of the reaction period, samples were centrifuged for $15 \mathrm{~min}$ at $1,600 \mathrm{~g}$ and $800 \mu \mathrm{l}$ of TriZol reagent was added to each tube followed by vigorous vortexing to allow cellular lysis and stabilize released RNA. Samples were then stored at $-80^{\circ} \mathrm{C}$ and recovered for RNA extraction as necessary.

\section{Sample collection and processing}

Adult patients diagnosed with histological or cytological advanced or metastatic cancer involving the bone; able to safely donate approximately $100 \mathrm{ml}$ of blood over a 2 to 4 -week period; and not receiving any chemotherapy or radiotherapy while in the study were included [25]. A total number of 28 patients were included and wholeblood and plasma was used for this study. Ten healthy volunteers were used to draw blood for expression analysis, 5 each in Paxgene and Tempus tubes. Among cancer patients, the majority were females (75\%). The tumor type were breast cancer $(n=19$, all females), multiple myeloma ( $n=4,2$ males/2 females), prostate cancer $(n=4)$ and renal cell carcinoma ( $\mathrm{n}=1$, male). A written informed consent was obtained in accordance with federal, state and institutional guidelines. Details on this patient population have been published elsewhere [25]. Whole blood and serum samples were collected from patients with skeletal metastasis that were enrolled in a nondrug interventional study to 
Citation: Kadam S, Cleverly AL, Farmen M, Grondin J, Cox YI, et al. (2013) A Canonical Transforming Growth Factor Beta-Dependent Signaling Pathway is Present in Peripheral Blood Cells of Cancer Patients with Skeletal Metastasis. J Mol Biomark Diagn 4: 153. doi:10.4172/21559929.1000153

donate blood. Visits were scheduled between a minimum of $48 \mathrm{~h}$ and a maximum of 7 days apart. Patients had one venipuncture at each visit for a maximum of four visits. At visit one, blood samples were collected for TGF- $\beta 1$, Platelet Factor 4 (PF4), Multianalyte Immunoassay Panel (MAIP) and SMAD assay, which assessed phosphorylated SMAD (pSMAD) and total SMAD (tSMAD). PBMCs were obtained for baseline and ex-vivo assays to determine PSMAD and tSMAD expression as previously described $[25,26]$. Briefly, blood was drawn into three 8 -mlvacutainer CPT containing $1 \mathrm{ml}$ of $0.1 \mathrm{M}$ sodium citrate for the SMAD assay. Tubes were inverted five times and centrifuged at room temperature for $25 \mathrm{~min}$ in a VanGuard V6500 centrifuge (SmithKline Beecham, PA, USA) at 1,300 g and processed to measure pSMAD and tSMAD levels in PBMCs. PBMCs were stimulated with TGF- $\beta 1$ and the treated cells (both stimulated or unstimulated) were lysed and stored frozen at $-80^{\circ} \mathrm{C}$ prior to analysis of pSMAD and total SMAD by ELISA as described elsewhere [25]. Patient samples for RNA extraction and microarray analysis were collected in tempus tubes (ABI, Foster City, CA). A minimum volume of $3 \mathrm{ml}$ blood was drawn in the $10 \mathrm{ml}$ tempus tube containing $6 \mathrm{ml}$ Red Blood Cell (RBC) lysis and RNA preservative solution. The tubes were shaken vigorously and stored at $-20^{\circ} \mathrm{C}$ until RNA extraction. Whole blood for testing in the Multianalyte Immunoassay Panel (MAIP) was collected in sodium citrate tubes and centrifuged at $1,500 \mathrm{~g}$ for 15 minutes at $2-6^{\circ} \mathrm{C}$. The topmost plasma portion was removed and recentrifuged under the same conditions. The resultant plasma sample was stored at $-80^{\circ} \mathrm{C}$ until analysis.

\section{RNA microarray, multianalyte immunoassay panel (MAIP), pSMAD and TGF- $\beta 1$ analysis}

Total RNA for microarray analysis from PBMCs was isolated from TRIzol aliquots following manufacturer's instructions, and purified using an RNeasy kit (Qiagen Inc., Valencia, CA). RNA from whole blood collected in Tempus tubes was extracted using globin mRNA reduction kit (GLOBIN NcClear, ABI, Foster City, CA). RNA integrity and yield were assessed by absorbance at 260 and $280 \mathrm{~nm}$ and analysis with the Agilent RNA 6000 Nano LabChip (Agilent Technologies, Inc., Palo Alto, CA). $5 \mu \mathrm{g}$ of RNA with OD 260:280 ratios of 1.8 and clear 18S and $28 \mathrm{~S}$ ribosomal RNA bands on the Agilent chip (RIN $>6$ ) was processed for application to Affymetrix U133A human array chip. TGF- $\beta$ levels were measured using a commercially available ELISA (R\&D Systems, Minneapolis, MN, catalog \# DB100) per the manufacturer's protocol. Briefly, plasma samples underwent initial acidification/neutralization steps immediately prior to analysis to activate latent TGF- $\beta$ to an immunoreactive form, under conditions which have been shown to minimize re-association with TGF- $\beta$ binding proteins [26]. The ELISA assay utilized soluble TGF- $\beta$ RII coated on the ELISA plate for antigen capture, with detection via a HRP-linked polyclonal specific for TGF- $\beta 1$. Recombinant human TGF- $\beta 1$ (rhTGF- $\beta 1$ ) included in the kit was used as a standard. In addition to TGF- $\beta 1$, separate aliquots from these same plasma samples were also batch analyzed for additional markers of platelet activation, PF4 and Beta-Thromboglobulin (BTG) using commercial enzyme immunoassays (Asserachrom ${ }^{\oplus} \mathrm{PF} 4$ and Asserachrom ${ }^{\bullet}$ BTG, both from Diagnostica Stago, Asnieres-Sur-Siene, France). Plasma samples were tested at Rules-Based Medicine (Austin, TX) in their human antigen Multiple Antigenic Peptides (MAP) platform.

\section{Microarray data collection and statistical data analysis}

Gene expression values were generated using Affymetrix Microarray Analysis Suite MAS5 algorithm to adjust array signal normalization, background adjustment and probe set/signal summarization. Array data that passed acceptable QC metrics for percent present call, scale factor and false positives was $\log 2$ transformed. The $\log 2$ transformed data was then standardized at the probeset level to generate $\mathrm{Z}$-scores: $Z=\frac{\mathrm{x}-\overline{\mathrm{x}}}{\mathrm{s} / \sqrt{\mathrm{n}}}$ where $\mathrm{X}$ is the expression value, $\overline{\mathrm{X}}$ is the mean expression value, $S$ is the standard deviation, and $n$ represents the number of samples [27]. Standardization was performed separately for each probe set derived from ex-vivo PBMC dataset and the patient sample dataset. The array profile of processed gene signal values were clustered and converted to heat maps using Spot fire Decision Suite. Array profiles were also integrated using the Cytoscape software for biological network visualization and the accompanying plug-in, BiNGO was used to map statistically significant gene ontology using a p-value of 0.01 [28]. Expression levels are presented as normalized z-scores which are derived from averaged probe set signal values across probe sets and annotated genes altered under various experimental conditions and in the whole blood of disease samples. Since some genes are down regulated by TGF- $\beta 1$ stimulation, the sign on the $z$-score is reversed before the average is taken. This composite value is referred to as the expression index [28].

Pearson correlation and partial correlation was used to assess associations. To determine association with expression index of LY2157299 monohydrate reversed genes and plasma based proteins we adjusted the response variable to account for platelet effects using PF4 levels as previously described [29]. Differential expression between ex-vivo PBMC treated groups was assessed by random effects ANOVA (SAS Proc mixed). Significance of gene expression differences between TGF- $\beta 1$ stimulated and non-stimulated PBMCs was made by calculating the False Discovery Rate (FDR) at less than 0.1. Reversal of TGF- $\beta 1$ effects by LY2157299 monohydrate was judged by p-values less than 0.05 .

\section{Results}

\section{Development of a TGF- $\beta 1$-dependent expression signature with PBMCs from healthy donors}

Previous studies have confirmed that cell lines exposed to TGF- $\beta 1$ produce specific changes in the expression of genes associated with TGF- $\beta$ RI mediated signaling $[8,30,31]$. More recently Allendorph et al. [32] described engineered ligands and their ability to functionally dissect the diversity of the transforming growth factor receptor super family activated response. We reasoned that normal PBMCs exposed to TGF- $\beta 1$ might produce a similar measurable "canonical" change in global expression provided the collection and handling of whole blood and the separation of PBMCs was adequately controlled to minimize the release of platelet derived transforming growth factors. The addition of a specific inhibitor of TGF- $\beta$ RI dependent pathway signaling in such an ex-vivo model system would then allow characterization of the specific cytokine/inhibitor dependent expression response. A schematic of the experimental overview is represented in Figure 1. Using PBMCs from healthy donors we determined global expression patterns at TGF- $\beta 1$ levels ranging from $0-100 \mathrm{pM}$, with and without pre incubation with $5 \mu$ M LY2157299 monohydrate. Development of this ex-vivo PBMC stimulation assay, its ability to detect TGF- $\beta 1$ dependent response as measured by phosporylation of SMAD proteins and the corresponding inhibition with LY2157299 monohydrate has been previously reported [25]. Here we have examined corresponding changes in global gene expression and evaluated the potential for deriving a canonical expression profile that may serve as a diagnostic tool to detect the 
Citation: Kadam S, Cleverly AL, Farmen M, Grondin J, Cox YI, et al. (2013) A Canonical Transforming Growth Factor Beta-Dependent Signaling Pathway is Present in Peripheral Blood Cells of Cancer Patients with Skeletal Metastasis. J Mol Biomark Diagn 4: 153. doi:10.4172/21559929.1000153

Page 4 of 8

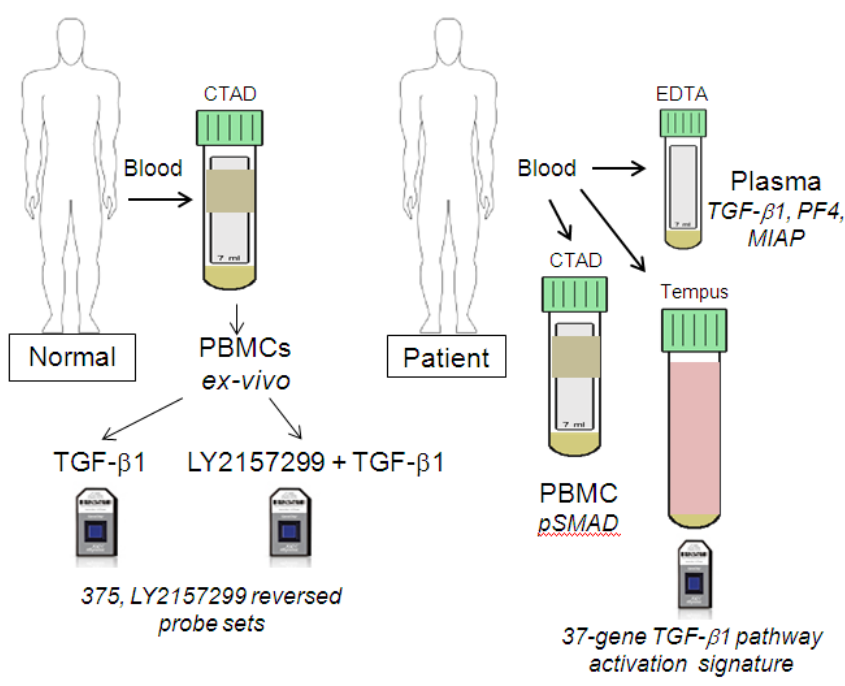

Figure 1: Schematic representation of samples and analysis performed on blood collected from normal subjects for ex-vivo analysis and cancer patients for measurement of global gene expression, pSMAD in PBMCs and plasma analytes. Blood collected in CTAD tubes was used to separate PBMCs for ex-vivo expression analysis and pSMAD measurement. Plasma from EDTA tubes was used for analysis of TGF- 31, PF4 and multiple analytes using the Multi Analyte Immunoassay Panel (MIAP). The Tempus blood collection tube was used for whole blood microarray analysis from patient samples.

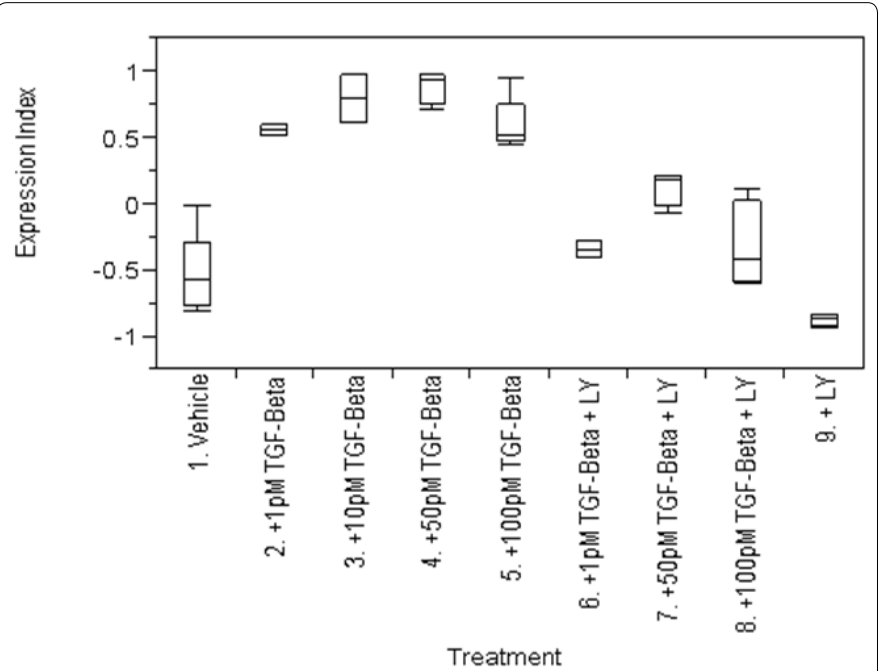

Figure 2: Distribution of the expression index values for 375 probe-sets in normal PBMC replicates treated with various levels of TGF- $\beta 1$ with and without $5 \mu \mathrm{m}$ LY2157299 monohydrate As described in materials and methods Lane (1) Vehicle represents no treatment and lane (9) represents LY 2159299 without TGF- $\beta 1$ addition. The box within each range of data points represents the upper and lower median of replicate values for each treatment group and line within the box represents the median value. The vertical line extending from the box where available represents the upper and lower range of data points for each experimental condition.

presence of TGF- $\beta 1$ dependent activation in blood from advanced cancer subjects.

The expression pattern of PBMCs stimulated with TGF- $\beta 1$ with and without LY2157299 monohydrate addition is represented in Figure 2. From left to right, the lanes (1-5) represent subgroups of samples with increasing TGF- $\beta 1$ levels followed by a subgroup (lanes 6-8) where $5 \mu \mathrm{M}$ LY2157299 monohydrate was added 30 min prior to addition of the various levels of TGF- $\beta 1$. Lane 9 represents baseline expression in normal PBMCs with $5 \mu \mathrm{M}$ LY2157299 monohydrate alone. Although samples without addition of the kinase inhibitor were exposed to TGF- $\beta 1$ for $4 \mathrm{~h}$ instead of $3.5 \mathrm{~h}$ the effect of the additional $30 \mathrm{~min}$ on expression was insignificant. There were 375 differentially expressed probe sets corresponding to 325 genes upon TGF- $\beta 1$ exposure (at all levels examined) that were reversed with LY2157299 monohydrate addition (LY-reversed). In addition 132 probe sets corresponding to 114 genes were differentially expressed with TGF- $\beta 1$ addition they were not reversed with LY2157299 monohydrate and were not included in further analysis (data not shown). The expression index calculated for the LYreversed 375 probe sets showed a clear difference with TGF- $\beta 1$ added compared with vehicle alone. There was a 2 -fold change in expression index median even at the lowest concentration of TGF- $\beta 1$ (1pM) that was almost completely reversed with the inhibitor. Interestingly, both with cytokine and with and without inhibitor addition, the pattern of the change became more diverse, with increasing TGF- $\beta 1$ concentration as more genes were activated perhaps as a result of non-specific TGF$\beta$ lactivity. This effect which underscores the sensitivity of TGF- $\beta$ signaling and the specificity of the expression index measure is evident in the width of the box that represents the upper and lower quartile in the box plot, Figure 2, lanes 2, 5 and 8 . At lowest level of TGF- $\beta$ tested activation and inhibition with LY2157299 is very sensitive and specific respectively, Figure 2 lanes 2 and 6 . In each case, when pre-incubated with the LY2157299 for $30 \mathrm{~min}$ the corresponding expression index of LY-reversed genes was reduced with TGF- $\beta 1$ addition. As expected, baseline LY-reversed expression index values were lowest and least variable with LY2157299 addition suggesting control of endogenous signaling from platelet-derived cytokines (see vehicle control).

\section{Development of a clinically relevant signature for systemic activation of the TGF- $\beta$ RI-dependent pathway}

We examined the ex-vivo 325 LY-reversed gene set for association with a comprehensive gene set consisting of 2,749 genes compiled by $\mathrm{Xu}$ and Kapoun [33] that was derived from several cell-lines treated with TGF- $\beta 1$. We found 113 common genes with a fold change range from 6.9 to -4.9 (data not shown). The expression of these genes was also altered in tumor specimens compared with corresponding normal tissue [33]. We rationalized that perhaps the effect of TGF- $\beta 1$ and altered expression in tissues might be similar in patient derived PBMCs where ligand was peripherally present. Since we had previously determined that ex-vivo PBMC activation with TGF- $\beta 1$ was also accompanied with accumulation of pSMAD [25], we hypothesized that residual phosphorylation capacity of SMAD proteins in PBMCs collected from cancer patients when stimulated with TGF- $\beta 1$ (ss) compared with unstimulated (us) would be reflected by the level of expression of TGF- $\beta 1$ specific genes in the PBMC sample. We therefore examined the 325 LY-reversed gene-set for genes with significant association with response to ex-vivo PSMAD stimulation from patients with advanced cancer to see if there was a systemic signature of TGF- $\beta 1$ activation.

The analytical measurement of ex-vivo pSMAD previously described [25] was derived from TGF- $\beta 1$ stimulated patient PBMCs and expressed as a ratio of stimulated (ss) versus unstimulated (su) ELISA signal values. We have previously demonstrated [26] that the pSMAD (ss/su) ratio was a reflection of the residual PBMC SMAD phosphorylation capacity and was inversely associated with plasma TGF- $\beta 1$ levels in these patients. Since we had collected whole blood from subjects and measured PBMC PSMAD stimulation, ex-vivo, using the same conditions as those used to derive the 325LY-reversed gene set, we were able to determined which subset from 325 LY-reversed 
Citation: Kadam S, Cleverly AL, Farmen M, Grondin J, Cox YI, et al. (2013) A Canonical Transforming Growth Factor Beta-Dependent Signaling Pathway is Present in Peripheral Blood Cells of Cancer Patients with Skeletal Metastasis. J Mol Biomark Diagn 4: 153. doi:10.4172/21559929.1000153

Page 5 of 8

genes also significantly correlated with plasma pSMAD (ss)/(su). We found that the expression index of 37 of 325 ex-vivo derived genes was significantly associated with patients' PBMC pSMAD (ss/su) response ratio, p-value $<0.0001$ as shown in Figure 3 where the dotted lines represent the confidence intervals around the simulated straight line correlation. We next examined the 37-gene expression index for association with plasma TGF- $\beta 1$ levels in patient samples and found

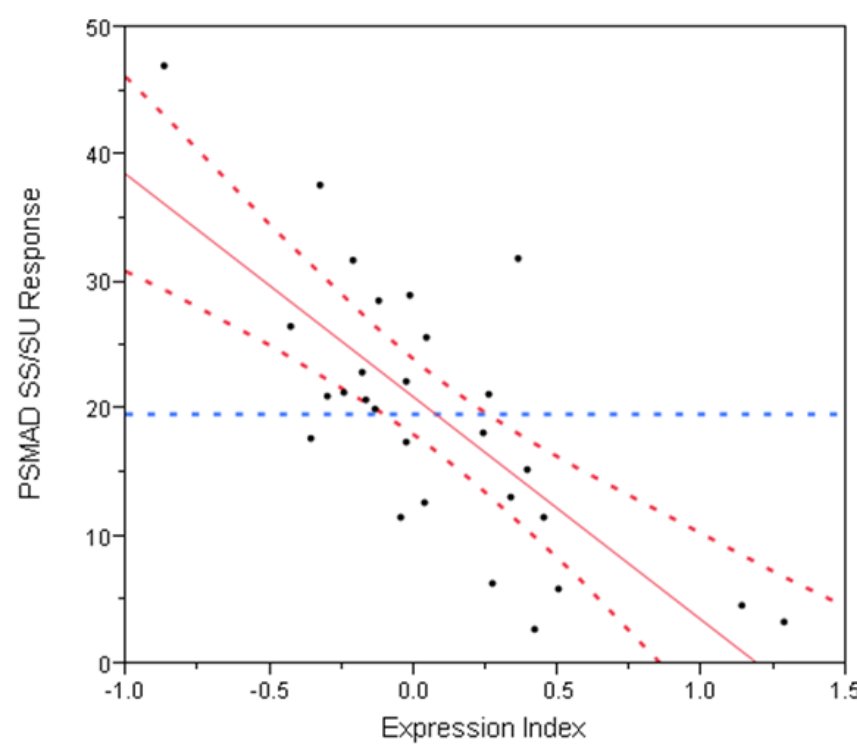

Figure 3: Association of pSMAD2 (ratio of TGF- $\beta 1$ stimulated and unstimulated) in patient PBMCs and the 37-gene whole blood Expression Index: Negative Correlation plot of the ratio of PBMC derived phosphorylated SMAD2/3, stimulated (SS)/unstimulated (SU)with the ex-vivo derived 37-gene expression index from whole blood of 28 cancer patients. The solid line represents the simulated correlation and the dotted lines represent the confidence interval. The horizontal dotted line intersects the area where the correlation was highest.

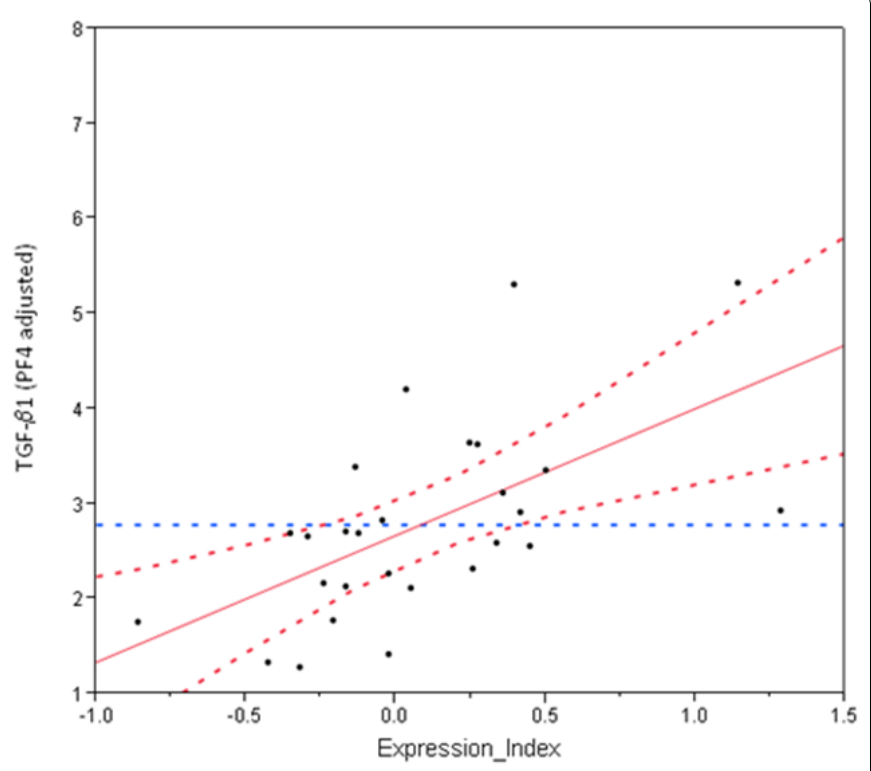

Figure 4: Positive Association of PF4 adjusted plasma TGF- $\beta 1$ level with the exvivo derived 37 -gene expression index from whole blood of 28 cancer patients. The simulated solid line represents the correlation and the surrounding dotted lines represent confidence interval. The horizontal dotted line intersects the area of highest correlation.

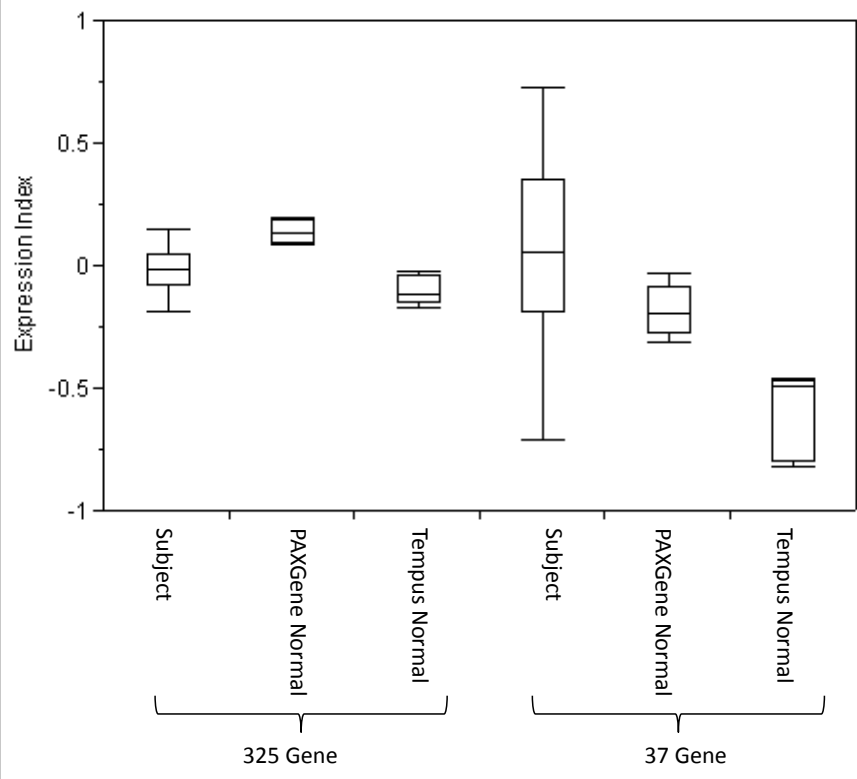

Figure 5: Representation of the expression index values for 375 probe-sets annotated to 325 genes and the reduced 37 -gene set in the whole blood from 28 cancer patients and 5 normal adults collected in PaxGene and Tempus RNA preservation tubes. Several replicated are represented in each experimental condition. The box within each range of data points represents the upper and lower median of replicate values for each treatment group and line within the box represents the median. The vertical line extending from the box where available represents the upper and lower range of data points for each experimental condition. The 37 -gene subset provided a broader dynamic range of expression index values compared with 325 -gene set.

that it was directly correlated with a p-value equal to 0.0014 (Figure 4). The statistically significant association of the ex-vivo derived 37-gene expression index with two separate known TGF- $\beta$ pathway activation measurements, plasma TGF- $\beta 1$ and PBMC pSMAD from clinical specimens further validates the 37 -gene signature as clinical "surrogate" for TGF- $\beta 1$ pathway activation.

\section{TGF- $\beta 1$ dependent gene profile is a surrogate for pathway activation in clinical specimens}

TGF- $\beta$ responsive changes in systemic expression have been previously reported in PBMCs $[8,30]$. However, affected cells were examined after separation from whole blood and cultured without adding TGF- $\beta$ raising the possibility of cellular re-equilibration and loss of the systemic effect resulting from chronic exposure to circulating TGF- $\beta$. We sought to examine whether there was evidence of systemic effect in patient blood by immediately accessing mRNA from affected cells upon immediate cell lysis and mRNA preservation. Since platelet-derived TGF- $\beta$ can confound the affect of circulating TGF- $\beta$ on WBC expression, we examined the effect of normal whole blood collected in a PaxGene tube where cells are stabilized in an RNA preserving buffer system without lysis and compared this with blood collected in a Tempus tube where cells are immediately lysed in a an RNA preservative containing lysis buffer. We then compared the distribution of the expression index values for the ex-vivo derived 325 gene LY-revered genes and the 37-gene PSMAD correlative TGF- $\beta 1$ responsive signature among Tempus tube collected patient blood and normal blood collected in PaxGene and Tempus tubes. As shown in Figure 5, the overall expression index for normal blood was higher in the PaxGene tubes compared with Tempus indicating that perhaps increased baseline expression in TGF- $\beta 1$ responsive genes within the 
Citation: Kadam S, Cleverly AL, Farmen M, Grondin J, Cox YI, et al. (2013) A Canonical Transforming Growth Factor Beta-Dependent Signaling Pathway is Present in Peripheral Blood Cells of Cancer Patients with Skeletal Metastasis. J Mol Biomark Diagn 4: 153. doi:10.4172/21559929.1000153

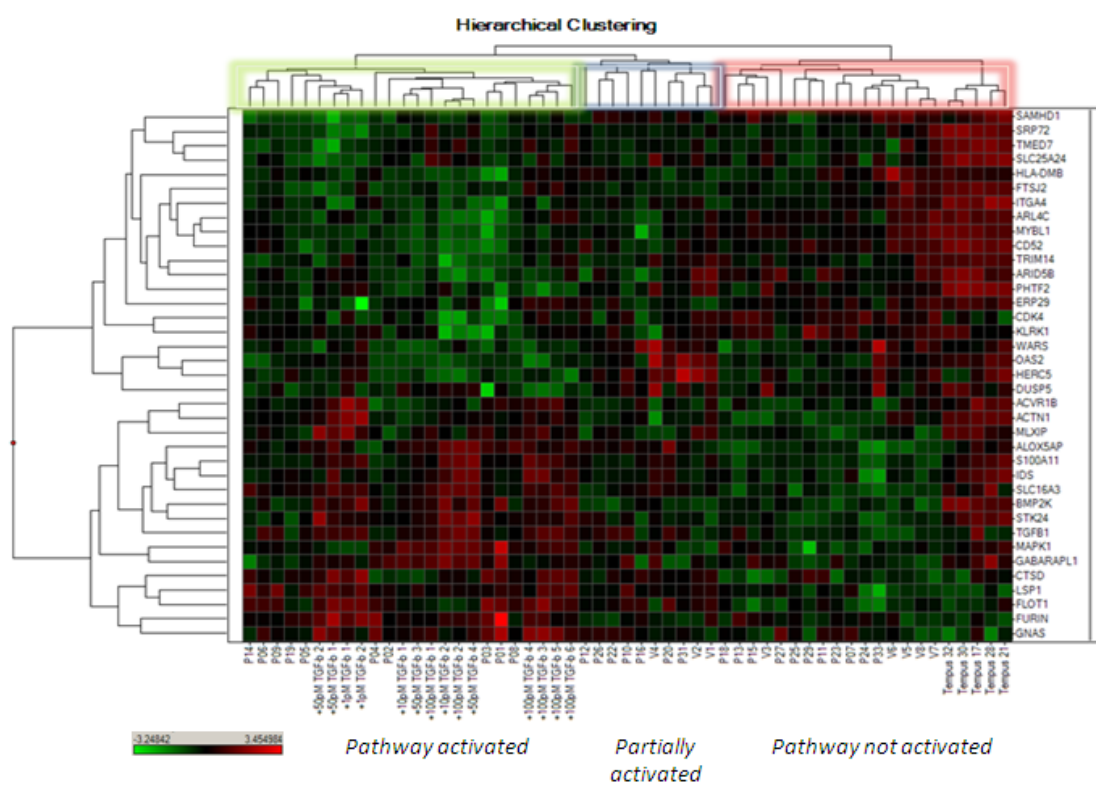

Figure 6: Hierarchically clustered heat map of the 37-gene expression signals from ex-vivo TGF- $\beta 1$ stimulated PBMCs, vehicle controls (PBMCs with no addition) and whole blood from 28 cancer patients and normal subjects. Two major clusters were seen, a TGF- $\beta$ PI pathway activated block to the left with all the ex-vivo TGF- $\beta 1$ stimulated samples and a block to the right with majority of normal (Tempus) and vehicle controls designated as pathway not-activated. A central block with intermediate expression was designated partially activated based on signal intensities.

\begin{tabular}{|l|l|l|l|}
\hline Protein & Gene Symbol/Probeset & Spearman R & p value \\
\hline ENA-78 & CXCL5/215101_s_at & 0.565 & 0.0049 \\
\hline Alpha-1-antitrypsin & Serpina1/2114974_s_at & 0.494 & 0.017 \\
\hline MMP-9 & MMP9/203936_s_at & 0.452 & 0.031 \\
\hline $\begin{array}{l}\text { Prostate Acid } \\
\text { Phosphatase }\end{array}$ & ACPP/204393_s_at & 0.451 & 0.031 \\
\hline Factor VII & F7/207300_s_at & 0.435 & 0.038 \\
\hline VEGF & VEGFA/210512_s_at & 0.431 & 0.036 \\
\hline EGF & EGF/206254_at & 0.402 & 0.058 \\
\hline Calcitonin & CALCA/210727_at & -0.367 & 0.085 \\
\hline TNF- $\beta$ & LTA/206975_at & -0.375 & 0.078 \\
\hline II-5 & IL5/207952_at & -0.395 & 0.062 \\
\hline PSA & KLK3/204582_s_at & -0.401 & 0.058 \\
\hline Erythropoietin & EPO/207257_at & -0.41 & 0.052 \\
\hline IL-3 & IL3/207906_at & -0.413 & 0.05 \\
\hline Lymphotactin & XCL1/206365_at & -0.415 & 0.049 \\
\hline Growth Hormone & GH1/211151_x_at & -0.429 & 0.041 \\
\hline
\end{tabular}

Abbreviations: ENA: Epithelial Cell-Derived Neutrophil-Activating Peptide; MMP9: Matrix Metallopeptidase-9; VEGF: Vascular Endothelial Growth Factor; EGF: Epidermal Growth Factor; TNF: Tumor Necrosis Factor; IL: Interleukin; PSA: Prostate Specific Antigen

Table 1: Association of secreted plasma protein levels with their corresponding gene expression signals in patient whole blood (cut-off $p<0.1$, unadjusted for multiplicity).

period of time it took to collection and preserve cells in this tube type. When the expression signal values for 37 genes TGF- $\beta 1$ responsive signature from 28 patient blood samples were analyzed together with ex vivo PBMC data from normal and stimulated samples the resulting hierarchical heat map showed three main clusters as shown in Figure 6. A cluster that separated with normal samples collected in Tempus tubes and ex vivo PBMC vehicle controls that included 11 patient samples which we have termed "pathway not activated". A cluster of 7 patient samples and 3 ex vivo PBMC vehicle controls termed "partially activated" and cluster with 10 patient samples that included all of the ex vivo PBMC samples with TGF- $\beta 1$ addition which was termed "pathway activated". Interestingly, we observed that the TGF- $\beta 1$ gene expression was always elevated with TGF- $\beta 1$ stimulated normal PBMCs but only sporadically in patient samples (Figure 6).

\section{Analysis of cytokine stimulated serum markers using a Multianalyte Immunoassay Panel (MAIP)}

Since a major function of TGF- $\beta 1$ appears to be the regulation of chemokine expression, we also measured several peripheral proteins known to be co-regulated with TGF- $\beta 1$. The values from the MAIP protein measurements were tested for correlation with the corresponding gene probe set signals present on the Affymetrix U133 gene chip and the results are depicted in Table 1. Several cytokines appeared to be significantly correlated, both positively and negatively in this analysis. Based on this finding, we asked whether the elevated TGF- $\beta 1$ in plasma was triggering the TGF- $\beta$ RI signaling pathway in patient PBMCs. Also given that the correlation of MAIP and gene expression might be related to the tumor histology and influence its development, we evaluated whether there were specific correlations between gene expression and certain proteins represented in the MAIP. Such a correlation between gene expression in the blood and plasma protein levels may help to find new markers of response to TGF- $\beta$ RI inhibitors. Hence, the correlation between ENA-78 and CXCL5/215101_s_at, VEGF and VEGFA/210512_s_at, as well as the correlation between MMP-9 and MMP9/203936_s_at, are intriguing observations for evaluation as potential predictive biomarkers of response in patient plasma.

\section{Discussion}

Recent progress in molecular oncology has provided increased optimism that empirical use of anticancer agents will soon be transformed into a practice of "personalized" cancer medicine, where molecular profile together with biomarker guided assessment of cellular content in patient's tumor, rather than tissue type and population-based 
Citation: Kadam S, Cleverly AL, Farmen M, Grondin J, Cox YI, et al. (2013) A Canonical Transforming Growth Factor Beta-Dependent Signaling Pathway is Present in Peripheral Blood Cells of Cancer Patients with Skeletal Metastasis. J Mol Biomark Diagn 4: 153. doi:10.4172/21559929.1000153

risk, will determine the choice of therapeutic intervention [32,33]. It has also become clear, that tumor response is guided by cellular molecular circuitry, in which these changes occur and are further facilitated by the presence of cytokines in the tumor microenvironment. This concept of context-dependent tumor addiction has important implications for the design of molecularly targeted therapies [34].

Although TGF- $\beta$ signaling is well known to play a key role in tumor progression and as a soluble mediator of immune regulation, the effects of the presence of this family of cytokines on peripheral cells in patient blood has not been clearly dissected. In cancer patients, secreted proteins are known to be released from various locations, such as tumor tissue, circulating blood cells and also from normal tissues, such as endothelial cells making it difficult to assess their functional effect in patients chronically exposed to circulating cytokines. Generally, only when tumors secrete either highly specific proteins or at high levels, does the assessment of their presence in the circulation become a meaningful laboratory measure for disease management. Paradoxically, TGF- $\beta$ isoforms are well known to act both as tumor suppressors and as tumor promoters and their subtle presence in the cellular environment can produce diverse outcomes including immune suppression and cellular senescence [35]. It has also been suggested that chronic exposure of tumors to TGF- $\beta$ might have an effect unlike acute exposure related expression changes derived from ex-vivo cell line based experiments and as such may require the analysis of both ex-vivo and patient derived specimens for biomarkers of TGF- $\beta$ response [31]. Since whole blood is readily accessible compared with tumor biopsies especially in advanced cancer patients, it is an ideal sample for assessment of TGF- $\beta$ dependent response and measurement of pharmacodynamic effect during the course of drug development. Additionally, PBMCs typically have a higher cellular turnover than resident tumor cells and thus a subset of the newly generated cells would be expected to be relatively naïve to the effects of continuous TGF- $\beta 1$ exposure unlike the primary tumor. These factors make whole blood an appropriate "surrogate" substrate for biomarkers of a systemic effect of TGF- $\beta 1$ induced RNA expression in cancer patients.

We used an ex-vivo assay with normal PBMCs suspended in a buffered matrix where the in-situ transcriptional capacity was fixed and then evaluated TGF- $\beta 1$-dependent mRNA expression as biomarker of its signaling activity. We then tested the PBMC based assay to determine the effect of addition of LY2157299 monohydrate, a small molecule inhibitor of the TGF- $\beta$ RI. First, we found that a specific set of transcripts was differentially regulated with and without addition of LY2157299 monohydrate prior to stimulation with TGF- $\beta 1$. Second, the pattern of change with inhibitor added appeared to mirror the effect seen when increasing levels of TGF- $\beta 1$ suggesting that perhaps the broader response seen at higher levels of TGF- $\beta 1(100 \mathrm{pM})$, resulted from significant saturation of cellular receptors, overwhelming specific TGF- $\beta 1$ dependent transcriptional capacity and allowing nonspecific TGF- $\beta 1$ derived expression causing a dilution of the specific response seen when the ligand was added at lower levels. This can become important when detecting TGF- $\beta$ 1gene signaling where there is too much circulating ligand, consequently making it harder to detect changes with LY2157299 treatment. Third, the ex-vivo assay was able to detect pre-analytical variability, due to suboptimal sample handling, known to set off release of platelet derived cytokines producing higher background expression prior to addition of exogenous TGF- $\beta 1$ and the resulting loss of LY2157999 monohydrate dependent reversal. This is also evident in the difference in the expression between the control (vehicle) and the inhibitor-only lanes Figure 2. Gene ontology analysis of the two gene profiles indicated that LY2157299 monohydrate specifically and significantly affected ligand induced genes in both directions where as changes in the non reversible gene set were less significant and nonspecific (data not shown). The non-reversed genes were annotated to know genes with cellular "housekeeping" function such as ACTB and GAPDH and thus, the resulting expression index might reflect cellular transcription capacity.

There are conflicting reports of the association of TGF- $\beta 1$ dependent expression in disease tissue, circulating levels and its effect on peripheral cells. For example, it was recently reported that in multiple sclerosis patients, while there was decreased activation of TGF- $\beta 1$ pathway signaling in whole blood and a reduction in the level of regulated genes, peripheral cells from these subjects (ex-vivo) retained an intact exogenous TGF- $\beta 1$ response [36]. Our data similarly shows a relationship between pSMAD response and the amount of TGF- $\beta 1$ stimulation. Since the tSMAD for paired unstimulated and stimulated PBMCs is strongly correlated, adjusting pSMAD for tSMAD in these samples was not necessary. However, since TGF- $\beta 1$ is a potent signaling molecule, the magnitude of transcriptional regulation from chronic exposure to TGF- $\beta 1$ is likely to be tightly controlled due to pleiotropic effects of this ligand. This may also explain the lack of a stronger correlation of the ex-vivo derived, LY2157299 monohydrate reversible expression index with peripheral TGF- $\beta 1$ levels in patient samples. However, when the reversible expression index was adjusted for PF4, there was good correlation with plasma TGF- $\beta 1$ levels, again suggesting that perhaps a naive subset of peripheral cells was TGF- $\beta 1$ responsive. Also, the correlation between pSMAD levels and gene expression suggests that the systemic 37 -gene TGF- $\beta 1$ responsive signature will be particularly important in conditions where TGF- $\beta$ signaling is partially activated. Additional support for the association of the LY2157299 monohydrate reversible expression index with TGF- $\beta 1$ was provided with the detection of co-regulated plasma derived proteins in patients. We found good overall correlation with multiple known co-regulated markers of TGF- $\beta 1$ activity listed in Table 1 . Specifically ENA-78, the protein product of CXCL5, MMP9 and VEGF are previously reported in animal models and tumor tissue to be tightly associated and prognostic of disease outcome in breast cancer [37]. Similarly, we found other known compensatory proteins such as EPO, TNF- $\beta$ and IL-3 to be negatively correlated with plasma TGF- $\beta$. This observation of correlation between blood-based proteins and gene expression in PBMCs further underscores that TGF- $\beta$ signaling is closely regulated in circulation. Additionally we noticed that based on microarray signal intensities the expression level of TGFB1 was higher in TGF$\beta 1$ stimulated PBMCs but only rarely in patient blood. It is worth noting that while the ex-vivo system provided a measure of change in expression that was clearly associated with known cytokine dependent changes in patient blood, the statistically significant observations reported here and the 37-gene TGF- $\beta 1$ expression signature came from 28 patient sample of which 19 were diagnosed with breast cancer. It is likely that length and type of standard of care treatment in patients may influence global expression in patient PBMCs. The unequivocal association of the 37-gene expression index as a "surrogate" gene expression signature for TGF- $\beta 1$ activation in tumors will require addition confirmation with robust clinical sampling. As drug development shifts towards target-based therapies [38] and the molecular assessment of disease subtypes becomes common place, the methodology described here in combination with specific inhibitors may become useful in disease diagnosis and the selection of appropriate treatment. Since TGF- $\beta$ RI signaling is known to be elevated in patients with bone metastasis [39], this study illustrates an approach using an expression profile from peripheral cells to potentially identify patients with an active TGF- $\beta$ signaling pathway supporting the disease condition $[40,41]$. 
Citation: Kadam S, Cleverly AL, Farmen M, Grondin J, Cox YI, et al. (2013) A Canonical Transforming Growth Factor Beta-Dependent Signaling Pathway is Present in Peripheral Blood Cells of Cancer Patients with Skeletal Metastasis. J Mol Biomark Diagn 4: 153. doi:10.4172/21559929.1000153

\section{Acknowledgements}

The authors thank colleagues in the Laboratory of Experimental Medicine for valuable technical input. Special thanks to Crystal Dotson for technical support and Lalit Verma for providing access to biological network maps and gene ontology tools. We also thank Dr. Smita Agarwal, for writing assistance provided with the manuscript.

\section{References}

1. Roberts AB, Flanders KC, Heine UI, Jakowlew S, Kondaiah P, et al. (1990) Transforming growth factor-beta: multifunctional regulator of differentiation and development. Philos Trans R Soc Lond B Biol Sci 327: 145-154.

2. Akhurst RJ, Derynck R (2001) TGF-beta signaling in cancer--a double-edged sword. Trends Cell Biol 11: S44-51.

3. Shim KS, Kim KH, Han WS, Park EB (1999) Elevated serum levels of transforming growth factor-beta1 in patients with colorectal carcinoma: its association with tumor progression and its significant decrease after curative surgical resection. Cancer 85: 554-561.

4. Dong ZZ, Yao DF, Yao M, Qiu LW, Zong L, et al. (2008) Clinical impact of plasma TGF-beta1 and circulating TGF-beta1 mRNA in diagnosis of hepatocellular carcinoma. Hepatobiliary Pancreat Dis Int 7: 288-295.

5. Hata A, Shi Y, Massagué J (1998) TGF-beta signaling and cancer: structural and functional consequences of mutations in Smads. Mol Med Today 4: 257 262

6. Mu Y, Gudey SK, Landstrom M (2011) Non-Smad signaling pathways. Cell Tissue Res 2011(epub ahead of print)

7. Baselga J, Rothenberg ML, Tabernero J, Seoane J, Daly T, et al. (2008) TGF-beta signalling-related markers in cancer patients with bone metastasis. Biomarkers 13: 217-236.

8. Classen S, Muth C, Debey-Pascher S, Eggle D, Beyer M, et al. (2010) Application of $\mathrm{T}$ cell-based transcriptomics to identify three candidate biomarkers for monitoring anti-TGFbetaR therapy. Pharmacogenet Genomics 20: 147-156

9. O'Brien PJ, Ramanathan R, Yingling JM, Baselga J, Rothenberg ML, et al. (2008) Analysis and variability of TGFbeta measurements in cancer patients with skeletal metastases. Biologics 2: 563-569.

10. Bueno L, de Alwis DP, Pitou C, Yingling J, Lahn M, et al. (2008) Semimechanistic modelling of the tumour growth inhibitory effects of LY2157299, a new type I receptor TGF-beta kinase antagonist, in mice. Eur J Cancer 44 $142-150$

11. Bomprezzi R, Ringnér M, Kim S, Bittner ML, Khan J, et al. (2003) Gene expression profile in multiple sclerosis patients and healthy controls: identifying pathways relevant to disease.Hum Mol Genet 12: 2191-2199.

12. Kerr JR, Petty R, Burke B, Gough J, Fear D, et al. (2008) Gene expression subtypes in patients with chronic fatigue syndrome/myalgic encephalomyelitis. $J$ Infect Dis 197: 1171-1184

13. Raghavachari N, Xu X, Munson PJ, Gladwin MT (2009) Characterization of whole blood gene expression profiles as a sequel to globin mRNA reduction in patients with sickle cell disease. PLoS One 4: e6484.

14. Segman RH, Shefi N, Goltser-Dubner T, Friedman N, Kaminski N, et al. (2005) Peripheral blood mononuclear cell gene expression profiles identify emergent post-traumatic stress disorder among trauma survivors. Mol Psychiatry 10: 500-513, 425.

15. Borovecki F, Lovrecic L, Zhou J, Jeong H, Then F, et al. (2005) Genomewide expression profiling of human blood reveals biomarkers for Huntington's disease. ProcNatIAcadSci 102: 11023-11028.

16. Wang Z, Beach D, Su L, Zhai R, Christiani DC (2008) A genome-wide expression analysis in blood identifies pre-elafin as a biomarker in ARDS. Am J Respir Cell Mol Biol 38: 724-732.

17. Asare AL, Kolchinsky SA, Gao Z, Wang R, Raddassi K, et al. (2008) Differential gene expression profiles are dependent upon method of peripheral blood collection and RNA isolation. BMC Genomics 9: 474

18. Robison EH, Mondala TS, Williams AR, Head SR, Salomon DR, et al. (2009) Whole genome transcript profiling from fingerstick blood samples: a comparison and feasibility study. BMC Genomics 10: 617.

19. Daly TM, Dumaual CM, Dotson CA, Farmen MW, Kadam SK, et al. (2005)
Precision profiling and components of variability analysis for Affymetrix microarray assays run in a clinical context. J Mol Diagn 7: 404-412.

20. Charles M (2010) Perou and Anne-LiseBørresen-Dale, Cold Spring HarbPerspectBiol 10: 1101

21. Gevensleben H, Göhring UJ, Büttner R, Heukamp LC, Kunz G, et al. (2010) Comparison of MammaPrint and TargetPrint results with clinical parameters in German patients with early stage breast cancer. Int J Mol Med 26: 837-843.

22. Misquitta-Ali CM, Cheng E, O'Hanlon D, Liu N, McGlade CJ, et al. (2011) Global profiling and molecular characterization of alternative splicing events misregulated in lung cancer. Mol Cell Biol 31: 138-150.

23. Sawyer JS, Anderson BD, Beight DW, Campbell RM, Jones ML, et al. (2003) Synthesis and activity of new aryl- and heteroaryl-substituted pyrazole inhibitors of the transforming growth factor-beta type I receptor kinase domain. J Med Chem 46: 3953-3956.

24. Sawyer JS, Beight DW, Britt KS, Anderson BD, Campbell RM, et al. (2004) Synthesis and activity of new aryl- and heteroaryl-substituted 5,6-dihydro-4Hpyrrolo[1,2-b]pyrazole inhibitors of the transforming growth factor-beta type I receptor kinase domain. Bioorg Med Chem Lett 14: 3581-3584.

25. Farrington DL, Yingling JM, Fill JA, Yan L, Qian YW, et al. (2007) Development and validation of a phosphorylated SMAD ex vivo stimulation assay. Biomarkers 12: $313-330$

26. Kropf J, Schurek JO, Wollner A, Gressner AM (1997) Immunologica measurement of transforming growth factor-beta 1 (TGF-beta1) in blood; assay development and comparison. Clin Chem 43: 1965-1974.

27. West M, Blanchette C, Dressman H, Huang E, Ishida S, et al. (2001) Predicting the clinical status of human breast cancer by using gene expression profiles Proc Natl Acad Sci U S A 98: 11462-11467.

28. Tibshirani R, Hastie T, Narasimhan B, Chu G (2002) Diagnosis of multiple cancer types by shrunken centroids of gene expression. Proc Natl Acad Sci U S A 99: 6567-6572.

29. Sall J (1990) Leverage Plots for General Linear Hypothesis. The American Statistician 44: 308-315

30. Sargent JL, Milano A, Bhattacharyya S, Varga J, Connolly MK, et al. (2010) A TGFbeta-responsive gene signature is associated with a subset of diffuse scleroderma with increased disease severity. J Invest Dermatol 130: 694-705.

31. Arteaga CL (2006) Inhibition of TGFbeta signaling in cancer therapy. Curr Opin Genet Dev 16: 30-37.

32. Allendorph GP, Read JD, Kawakami Y, Kelber JA, Isaacs MJ, et al. (2011) Designer TGF ${ }^{2}$ superfamily ligands with diversified functionality. PLoS One 6 e26402.

33. Xu XL, Kapoun AM (2009) Heterogeneous activation of the TGFbeta pathway in glioblastomas identified by gene expression-based classification using TGFbeta-responsive genes. J Transl Med 7: 12.

34. Varmus $\mathrm{H}$ (2006) The new era in cancer research. Science 312: 1162-1165.

35. Weinstein IB (2002) Cancer. Addiction to oncogenes--the Achilles heal of cancer. Science 297: 63-64.

36. Felsher DW (2010) MYC Inactivation Elicits Oncogene Addiction through Both Tumor Cell-Intrinsic and Host-Dependent Mechanisms. Genes Cancer 1: 597 604

37. van Riggelen J, Müller J, Otto T, Beuger V, Yetil A, et al. (2010) The interaction between Myc and Miz1 is required to antagonize TGFbeta-dependent autocrine signaling during lymphoma formation and maintenance. Genes Dev 24: 12811294.

38. Meoli EM, Oh U, Grant CW, Jacobson S (2011) TGF-î́2 signaling is altered in the peripheral blood of subjects with multiple sclerosis. J Neuroimmunol 230 : 164-168.

39. Bierie B, Chung CH, Parker JS, Stover DG, Cheng N, et al. (2009) Abrogation of TGF-beta signaling enhances chemokine production and correlates with prognosis in human breast cancer. J Clin Invest 119: 1571-1582.

40. Fox E, Curt GA, Balis FM (2002) Clinical trial design for target-based therapy. Oncologist 7: 401-409.

41. Kang Y, He W, Tulley S, Gupta GP, Serganova I, et al. (2005) Breast cancer bone metastasis mediated by the Smad tumor suppressor pathway. Proc Natl Acad Sci U S A 102: 13909-13914. 